\title{
A theoretical review on the origin of medicinal practices in humans: echoes from evolution
}

Washington Soares Ferreira Júnior ${ }^{1, *}$ and Ulysses Paulino Albuquerque ${ }^{2}$

\begin{abstract}
Humans have been coping with diseases throughout the evolutionary history. The emergence of medical systems, which integrate knowledge and practices related to health management, can be assumed as one of the reactions of the human species to respond to the pressures of diseases. An intriguing question is related to the understanding of how these systems have emerged in our species. To answer this question, some authors created scenarios to explain the origin of these systems, from an ecological and evolutionary logic. In this article, we briefly introduce some of the key ideas linked to these scenarios and how they may be important in guiding ethnobiology research.
\end{abstract}

Keywords: Evolutionary Ethnobiology; Medical Systems; Chemical Ecology; Medicinal Plants.

\footnotetext{
1 Universidade de Pernambuco, BR 203, Km 2, S/N, Cidade Universitária, Petrolina, Pernambuco, Brazil, 56.328-903.

${ }^{2}$ Laboratório de Ecologia e Evolução de Sistemas Socioecológicos, Centro de Biociências, Departamento de Botânica, Universidade Federal de Pernambuco, Cidade Universitária, Recife, Brazil, 50.670-901.

* Corresponding author. $\mathrm{E}$ E-mail address: WSFJ (washington.ferreira@upe.br), UPA (upa677@hotmail.com).
}

\section{INTRODUCTION}

Our species has been exposed to different disease conditions throughout its evolutionary history. Changes in health conditions may be linked to different factors, such as environmental, genetic and cultural (Dunn 1976). In order to cope with this, human beings have developed medical systems, which allow a link between concepts and practices in disease prevention and treatment (Jain and Agrawal 2005). Particularly, the medical system represents the set of knowledge and practices involving (1) the identification of diseases, aggregating knowledge about the causes of diseases and experiences of recognized symptoms; (2) disease-related behaviors and strategies and alternatives for treatment, such as plant and animal species, among other alternatives; (3) and the evaluation of therapeutic results. Thus, changes in the components mentioned above in different human groups may also indicate different medical systems (Kleinman 1978). 
The concepts of health and disease and treatment strategies may be different in the same human group, particularly in three social sectors, namely: (a) "popular sector"; (B) "professional sector" and (c) "folk sector" (Kleinman 1978). The "popular sector" includes decision-making and knowledge about diseases in the popular domain, in the family context. The "folk sector" includes local non-professional specialists recognized by the local culture as knowledgeable and holders of the knowledge about health, disease and treatments of the local system, such as folk healers, midwives, etc. In the "professional sector" are included all professionals of scientific medicine and professionalized traditions such as the Chinese and ayurvedic, for example (Kleinman 1978). Each of these sectors can have its own body of disease concepts and directions for a particular treatment.

In this sense, human beings present a great diversity of concepts about health and disease, and can attribute different causes to health problems (Hodes 1997; Levin and Browner 2005). For instance, there are communities in India that attribute health problems to deviations from customs and social norms, taboo breaks and the punishment of evil spirits (Mukherjee 2003). We can thus question ourselves about the role that medical systems have played in the survival of the human species. Considering that diseases have been an important selective force for human evolution, medical systems can be thought of as a response of our species to minimize health problems (Brown 1987). Some scientists have been thinking about this, particularly in understanding how these systems originated and how they evolve over time. In this article, we present three of the main theoretical scenarios, which are based on assumptions of ecology and evolution, which seek to explain the origin and evolution of these systems. These scenarios can help us understand the possible role of medical systems in human evolution.

\section{The origin and evolution of medical systems}

Some authors are prominent in the effort to understand the origin and evolution of medical systems, such as Horacio Fabrega Jr. (Fabrega 1997), Timothy Johns (Johns 1990) and Benjamin Hart (Hart 2005). Chronologically, T. Johns published his book in 1990, which launched a theoretical scenario based on chemical ecology ${ }^{1}$ to explain the origin and evolution of medical systems. H. Fabrega Jr., influenced by the ideas of $\mathrm{T}$. Johns, presents an evolutionary scenario associated with the emergence and development of medical systems. Finally, Benjamin Hart discusses some hypotheses connected with the origin of medicine. Here we present some of the main ideas of these authors.

\section{The hypotheses of learning and natural selection}

One of the interesting models to understand the origin of medicine involves the study of the use of medicinal plants in various human groups. According to B. Hart, there are two possible hypotheses for the

\footnotetext{
${ }^{1}$ The chemical ecology seeks to understand how the interactions between individuals of the same or different species can be chemically mediated, such as the presence of pheromones that favor communication between individuals (see Mori 2013).
} 
origin of the use of plants for the treatment of diseases in the early stages of development of medical systems: the hypothesis of learning and the hypothesis of natural selection. The idea of learning proposes that the origin of medicine is based on the processes of association that may have occurred in the past, in which the individual associated the ingestion of a plant with the relief of diseases symptoms, such as the relief of pain and malaise. Once the symptoms returned, the individual could remember the previous use and again consume the plant that relieved the symptoms. In this sense, a number of plants would be associated with the relief of disease symptoms and this information would be passed on by social transmission to other individuals, forming what would be a version of the early medical systems. The idea of learning relates not only to the experiences that humans had of experimenting with plants in the early stages, but also to observing animal behavior. Possibly, by observing the behavior of animals, the first humans have avoided the consumption of toxic plants.

The hypothesis based on natural selection suggests a different situation, indicating that the origin of medical systems is not necessarily based on learning. From this explanation, individuals are born with a behavior of ingesting certain plants when ill, and these selected plants would have particular characteristics. For example, ill individuals in the evolutionary past could be selecting plants based on bitter and astringent taste, which would lead the individual to consume the part of the plant in which these markers were present. This behavior would not be associated with any previous experience, but because the individual preferred to ingest bitter resources when ill. In that case, this preference would be innate. Based on this explanation, in the evolutionary past, it would be possible to suppose that diseased individuals who had this behavioral predisposition could increase their survival and reproduction in relation to the diseased individuals without this predisposition.

Some studies show the importance of bitter taste in the medicinal use of plants in different medical systems (Brett 1998; Molares and Ladio 2009; Medeiros et al. 2015a). This may suggest that the taste marker has a relevant role in the selection of plants for medicinal use (see Medeiros et al. 2015b). Some evidence suggests that compounds recognized as bitter may have important pharmacological actions (see, for example, Mennella et al. 2013). However, robust evidence is still lacking to associate the current importance of bitter taste with the possible explanations of learning and natural selection for the origin of medicine.

\section{The sickness-healing adaptation}

H. Fabrega argues that the disease affects the ability of individuals to care for their relatives, also affecting the reproduction and social functioning of human groups. The evolution of the social behavior of self care and the care of the other has a great importance in human evolution (Fabrega 1997). The development of higher nervous systems in animals favored the recognition of changes in the conditions of the own body and also in the perception of behavior change of other individuals, which may have provided one of the basis for the specialization of caring behaviors.

The perception of changes in one's body and other organisms associated with disease conditions may have been a selected behavior throughout animal evolution. In humans, the habits to socially 
recognize and share information about diseases and their treatment provided a significant leap in human evolution. Moreover, it shows a relationship between biology and culture. It is in this sense that Fabrega (1997) argues that the origins of medical systems had a biological basis and were improved from the interaction between biology and culture. The author indicates that the recognition of diseases and the treatment employed are rooted in the same adaptation (sickness-healing adaptation - SH adaptation), being an innate behavior of humans about the self care and the care of the other. This means that individuals are already born with a predisposition to caring behavior linked to disease events.

Fabrega (1997) suggests that the evolutionary pressures that led to the selection of disease recognition adaptation and treatment (SH adaptation) were, for example, competition between early hominids from different groups, foraging activities such as hunting practices, and the presence of predators, which increased the number of injuries and diseases, creating the favorable scenario for the selection of $\mathrm{SH}$ adaptation. This adaptation allowed the benefit of sick organisms in a group to be protected and rehabilitated, leading to group maintenance and social reciprocity (Fabrega 1997).

Figure 1 represents the model of a medical system in an evolutionary scenario, as proposed by Fabrega (1997). In this model, the three initial circles (from the center) indicate the basic nucleus of a medical system and refers to the level of individuals, compounds of medical genes, medical memes ${ }^{2}$ and phenotypic products.
Medical genes refer to the basic genetic units present in the medical system, being the genetic information units that are associated with $\mathrm{SH}$ adaptation, such as the sensory units that assist in the perception of the disease and the innate behavior of care. Medical memes are the cultural information units that are involved in the system. New individuals are not born with the knowledge of medical memes, such as naming disease a or b. However, people are born with a biological, innate predisposition to perceive diseases and later learn to name these diseases.

Thus, culture provides the context for innate behaviors to be expressed. The product of circles one and two is found in circle three, which is the response, the expression of behavior as a product of the interaction between genes and medical memes. Other systems may exert influence on the medical system, such as institutions or religious systems, and political-economic systems, as shown in Figure 1.

\section{The Human Chemical Ecology Model}

Timothy Johns focused his efforts on understanding the relationship between human groups and the diversity of chemical compounds in the environment (Johns 1990). Their questions have focused on understanding how different human groups have adapted to recognize and separate beneficial and toxic compounds from plants and animals. Johns argues that the early hominids were able to select environmental resources gaining a nutritional benefit and minimizing the side effects of ingestion of

\footnotetext{
2 We use the term "medical memes" in this text to preserve the way Fabrega (1997) developed his scenario for the evolution of medicine. However, we do not use the term "meme" here as an approximation of memetics, but only as units of cultural information that are learned by individuals. Memetics presents a set of definitions and explanations that we are not assuming in this text.
} 


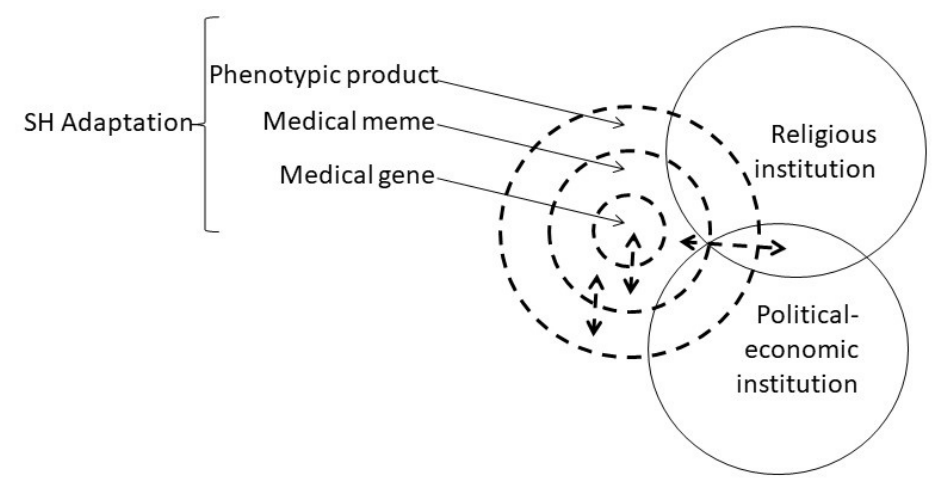

Figure 1. Model representing the elements of a medical system that constitute the adaptation of health care and treatment of diseases ( $\mathrm{SH}$ adaptation), composing the medical gene, medical meme and its phenotypic products. The two larger circles generate interacting forces with the medical elements, such as religious and political-economic systems and institutions. The arrows represent the interrelations between the medical elements and between the medical elements and the non-medical institutions. Adapted from Fabrega (1997).

parts of animals and plants for food. In this sense, the origin of human medicine may be related to a human adaptive response to effects caused by plant toxins ingested when feeding (Johns 1990).

When in contact with the compounds produced by plants, the first hominids had to deal with possible toxins that altered their health, involving a number of biological adaptations and also a number of cultural practices to minimize the effect of these toxins. For example, Johns (1990) interpreted taste and smell perception as a biological adaptation in the recognition of certain properties of compounds, as well as a behavioral predisposition to react to the perceived taste, such as the rejection of certain tastes that could concentrate toxic substances.

The development and transmission of practices to cope with toxic chemical compounds, such as the detoxification processes of plant parts for ingestion with the use of fire, also played a role in minimizing the effects of toxins (Johns 1990). From these considerations, the selection of plants in medical use in medical systems reflects an integrated response of human groups to the environment to which they are inserted. The assumptions of this model are presented below (see Figure 2):

1. "Humans seek to maintain
physiological homeostasis through
maximizing the beneficial effects of ingested
components and minimizing the effects of
potential toxins" (Johns 1990, p. 14).

2. "Natural selection has produced the interrelated physiological and behavioral mechanisms that allow humans to deal with environmental chemicals" (Johns 1990, p. 14).

3. "Humans have unique cultural traits that play a role in their interactions with plant (and animal) chemical constituents. Language and technological innovations, 
including plant domestication, are particularly powerful forces" (Johns 1990, p. 19).

4. "The nature of human interactions with plant (and animal) chemicals occurs within, and is influenced by, a broad ecological framework" (Johns 1990, p. 22).

Humans cope with plant chemical compounds through biological adaptations, based on physiological and behavioral adaptations, and also through cultural practices, the evolution of communication, learning and language processes, which communicate the practices for detoxification of parts of the plant. Plants also undergo different pressures, which may lead to the selection of different chemical compounds that they produce (Johns 1990). Among these pressures, we can situate the selection and continuous management that humans have performed in certain plant populations, leading to domestication processes (Parra et al. 2012; Casas et al. 2015). This scenario supports coevolutionary relationships between human groups and the chemical compounds of plants (Figure 2).

\section{FINAL CONSIDERATIONS}

The three scenarios presented may provide signs about the paths that were traced by early hominids in the evolutionary past that led to the emergence of medical systems. The appreciation of these scenarios suggests that the emergence of these systems may have been mediated not only by biological factors but also by cultural factors. Particularly, interactions between biology and culture may be important in understanding the development of these systems, placing medical systems as biocultural entities. In addition, the scenarios discussed do not appear to be mutually exclusive. The three can complement each other in the comprehension of the origins of

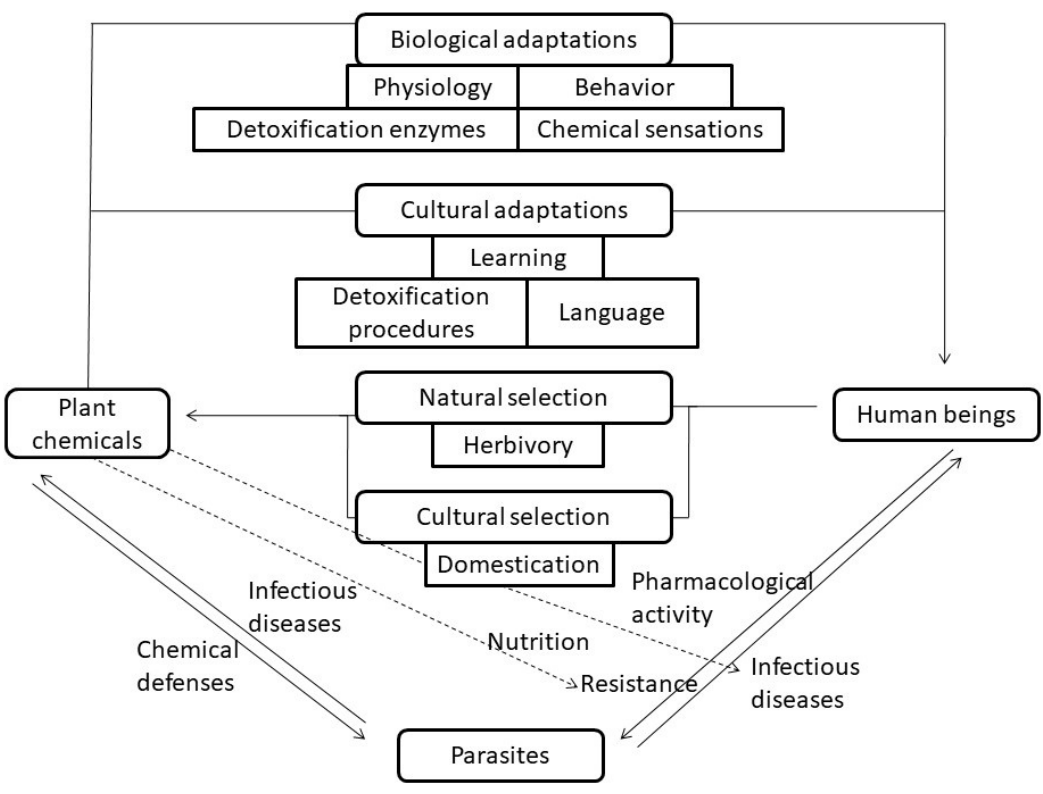

Figure 2. Human ecology model, according to Johns (1990). This model expresses the coevolutionary relations between human groups and chemical compounds of plants. 
medical systems. This could lead to future integrated research efforts to understand the role of learning, the evolution of care in relation to diseases, and the interactions of people with plant chemical compounds in the emergence of such systems.

\section{REFERENCES}

Brett JA (1998) Medicinal plant selection criteria: the cultural interpretation of chemical senses. Angewandte Botanik 72:70-74.

Brown PJ (1987) Microparasites and macroparasites. Cultural Anthropology 2:155171.

Casas A, Parra F, Blancas J (2015) Evolution of humans and by humans. In: Albuquerque UP, Medeiros PM, Casas A (eds) Evolutionary ethnobiology. Springer, New York, pp. 21-36.

Dunn F (1976) Traditional Asian medicine and cosmopolitan medicine as adaptive systems. In: Leslie C (ed) Asian medical systems: a comparative study. University of California Press, California, pp. 133-158.

Fabrega H (1997) Evolution of sickness and healing. University of California Press, Berkeley, USA.

Hart BL (2005) The evolution of herbal medicine: behavioural perspectives. Animal Behavior 70:975-989.

Hodes RM (1997) Cross-cultural medicine and diverse health beliefs. Ethiopians abroad. The Western Journal of Medicine 166:29-36.

Jain S, Agrawal S (2005) Perception of illness and health care among Bhils: a study of Udaipur District in Southern Rajasthan. Studies Tribes and Tribals 3:15-19.
Johns T (1990) With bitter herbs they shall eat it: chemical ecology and the origins of human diet and medicine. University of Arizona Press, Tucson, USA.

Kleinman A (1978) Concepts and a model for the comparison of medical systems as cultural systems. Social Science \& Medicine 12:85-93.

Levin BW, Browner CH (2005) The social production of health: critical contributions from evolutionary, biological, and cultural anthropology. Social Science \& Medicine 61:745-750.

Medeiros PM, Ladio AH, Albuquerque UP (2015a) Local criteria for medicinal plant selection. In: Albuquerque UP, Medeiros PM, Casas A (eds) Evolutionary ethnobiology. Springer, New York, pp. 149-162.

Medeiros PM, Santos Pinto BL, Nascimento VT (2015b) Can organoleptic properties explain the differential use of medicinal plants? Evidence from Northeastern Brazil. Journal of Ethnopharmacology 159:43-48.

Mennella A, Spector A, Reed D, Coldwell SE (2013) The bad taste of medicines: overview of basic research on bitter taste. Clinical Therapy 35:1225-1246.

Molares S, Ladio A (2009) Chemosensory perception and medicinal plants for digestive ailments in a Mapuche community in NW Patagonia, Argentina. Journal of Ethnopharmacology 123:397-406.

Mukherjee BM (2003) Cultural aspects of health in Jowhar of Maharashtra. Studies Tribes \& Tribals 1:163-164.

Parra F, Blancas JJ, Casas A (2012) Landscape management and domestication of Stenocereus pruinosus (Cactaceae) in the Tehuacán Valley: human guided section and gene flow. Journal of Ethnobiology and Ethnomedicine 8:32.

Received: 06 June 2017

Accepted: 02 Februay 2018

Published: 28 February 2018 